\title{
Novos agrotóxicos e o padrão de potabilidade da água: dinâmica ambiental e riscos à saúde
}

\author{
New pesticides and the drinking water standards: \\ environmental fate and health risks
}

Taciane de Oliveira Gomes de Assunção ${ }^{1}$ (B), Fernanda Bento Rosa Gomes ${ }^{1}$ (D), Emanuel Manfred Freire Brandt ${ }^{1}$ (D), Renata de Oliveira Pereira ${ }^{1}$ (D)

1Universidade Federal de Juiz de Fora - UFJF, Juiz de Fora, MG, Brasil. E-mails: taciane.assuncao@engenharia.ufjf.br, fernanda.bento@engenharia.ufjf.br, emanuel.brandt@ufjf.edu.br, renata.pereira@ufjf.edu.br

Como citar: Assunção, T. O. G., Gomes, F. B. R., Brandt, E. M. F., \& Pereira, R. O. (2020). Novos agrotóxicos e o padrão de potabilidade da água: dinâmica ambiental e riscos à saúde. Revista de Gestão de Água da América Latina, 17, e16. https://doi.org/10.21168/rega.v17e16

\begin{abstract}
RESUMO: Os agrotóxicos são amplamente utilizados no Brasil. Uma vez no ambiente podem contaminar o ar, a água e o solo e provocar danos à saúde dos seres humanos. Com a recorrente autorização de uso de diversos agrotóxicos no Brasil, a presente nota técnica tem por objetivo avaliar a dinâmica ambiental e a toxicidade dos ingredientes ativos de agrotóxicos que obtiveram seus registros aprovados pelo governo brasileiro no período de janeiro de 2018 a dezembro de 2019, bem como sugerir possíveis valores-normativos na água de consumo humano no Brasil. Dos 15 compostos autorizados no período, foram avaliados somente os ingredientes ativos de origem química (Dinotefuran, Florpirauxifen-benzil, Fluopiram e Sulfoxaflor), sendo excluídos da análise os compostos excipientes e agentes microbiológicos. Dinotefuran e Fluopiram destacaram-se por apresentar elevada toxicidade e persistência ambiental, o que pode indicar a possibilidade de exposição de seres humanos via consumo de água. O Sulfoxaflor, apesar de possuir dinâmica ambiental desfavorável à contaminação dos mananciais de abastecimento, requer um acompanhamento de sua ocorrência, devido à sua elevada toxicidade. Por fim, o Florpirauxifen-benzil não demonstrou risco que justifique seu monitoramento em mananciais.
\end{abstract}

Palavras-chave: Dinotefuran; Florpirauxifen-benzil; Fluopiram; Sulfoxaflor.

ABSTRACT: Pesticides are widely used in Brazil. Once in the environment, they can contaminate the air, the water and the soil, and they may cause harm to human health. With the recurrent approval of many registers of pesticides in Brazil, the aim of this paper is to evaluate the environmental dynamic and the toxicity of the active ingredients of pesticides that had their records approved from January 2018 to December 2019, along with a suggestion of possible guideline values for the equivalent concentration in Brazilian drinking water. Of the 15 compounds that were approved in the period, were selected only the chemical compounds (Dinotefuran, Florpyrauxifen-benzyl, Fluopyram, Sulfoxaflor), excipient ones and microbiological agents were excluded. Dinotefuran and Fluopyram stood out for presenting high toxicity and environmental persistence, which may indicate the possibility of exposure of human beings to high levels of these pesticides by water consumption. Sulfoxaflor, on the other hand, despite having unfavorable environmental dynamics to the contamination of water sources, requires monitoring of its occurrence in water supply systems, due to its high toxicity. Finally, Florpyrauxifen-benzyl has not shown evident risk that justifies its monitoring in water sources.

Keywords: Dinotefuran; Florpyrauxifen-benzyl; Fluopyram; Sulfoxaflor.

\section{INTRODUÇÃo}

Os agrotóxicos são substâncias e agentes físicos, químicos ou biológicos, utilizados para modificar a composição da flora e da fauna a fim de preservá-las da ação danosa de seres vivos considerados nocivos (Brasil, 1989). Esses compostos são empregados de maneira extensiva no Brasil (Pereira et al., 2019), principalmente pelo fato da atividade agrícola ser um processo produtivo de grande destaque na economia nacional (Veiga, 2007). Em contato com o ambiente, os agrotóxicos podem contaminar o ar, o solo e os mananciais superficiais e subterrâneos, expondo os seres humanos a riscos químicos associados principalmente à ingestão de água e alimentos contaminados. Diversos efeitos adversos à

Recebido: Julho 29, 2020. Revisado: Setembro 01, 2020. Aceito: Setembro 02, 2020. 
saúde humana podem ser destacados pela exposição crônica aos agrotóxicos: danos no fígado e nos sistemas nervoso central, reprodutivo e cardiovascular, podendo ampliar o risco de desenvolvimento de câncer, dentre outros efeitos adversos (Pereira et al., 2019; Agency for Toxic Substances and Disease Registry, 2019).

No Brasil, o Decreto $n^{\circ} 4.074$ de 22 de janeiro de 2002 regulamenta a Lei $n^{\circ} 7.802$ de 11 de julho de 1989 que dispõe sobre a experimentação, a pesquisa, o processo de fabricação até sua distribuição final, o registro, a classificação, a comercialização, a inspeção e a fiscalização de agrotóxicos e afins (Brasil, 1989), cabendo ao Ministério da Agricultura, Pecuária e Abastecimento (MAPA), ao Ministério do Meio Ambiente (MMA) e ao Ministério da Saúde (MS) a execução das prerrogativas estabelecidas. Ademais, segundo o artigo 2, parágrafo $3^{\circ}$ do Decreto $\mathrm{n}^{\circ} 4.074$, é de responsabilidade desses ministérios a determinação do limite máximo de resíduos (LMR) em alimentos e o intervalo de segurança dos agrotóxicos e afins. Além disso, é de responsabilidade dos mesmos ministérios a publicação no Diário Oficial da União do resumo dos pedidos e das concessões de registros no Brasil (Brasil, 2002). A autorização de uso de um ingrediente ativo (IA) no país implica na possibilidade de exposição da população via diferentes formas. Assim, deve-se realizar uma avaliação de risco dos agrotóxicos para garantir a utilização segura desse recurso. A metodologia de avaliação quantitativa de risco químico (AQRQ) é amplamente utilizada para esse propósito e pressupõe quatro etapas: identificação do perigo, avaliação da dose-resposta, avaliação da exposição e caracterização do risco (United States Environmental Protection Agency, 2020).

Nesse contexto, a compreensão da dinâmica ambiental dos agrotóxicos é de suma importância na avaliação da possibilidade de exposição, ou seja, do potencial de contaminação das diferentes matrizes (ar, solo, águas superficiais e/ou subterrâneas), bem como da persistência dessas substâncias no ambiente (Valarini et al., 1998). Paralelamente, os estudos toxicológicos auxiliam na avaliação da dose-reposta e, portanto, da magnitude do risco associado à exposição à tais agentes (Associação Brasileira de Saúde Coletiva, 2015). Com base em estudos de toxicidade e dinâmica ambiental, podem se pautar estratégias de minimização de impactos e riscos à saúde humana (Rice et al., 2007) ou medidas de restrição e proibição do uso (Agência Nacional de Vigilância Sanitária, 2019a).

A partir do conhecimento do risco associado à exposição crônica por agentes químicos via consumo de água (World Health Organization, 2017), diversos países e organizações estabeleceram valores-normativos/orientadores para a ocorrência de tais substâncias em água potável (Brasil, 2017; World Health Organization, 2017; Health Canada, 2017; National Health and Medical Research Council, 2018; New Zealand, 2018; United States Environmental Protection Agency, 2018a). No Brasil, a Portaria de Consolidação MS no 5/2017 (Anexo XX) dispõe sobre o padrão brasileiro de potabilidade da água para consumo humano e define os valores máximos permitidos (VMP) para as diversas características físicas, químicas e biológicas na água potável, incluindo 38 princípios ativos e metabólitos de agrotóxicos (Brasil, 2017). Além da toxicologia, a inclusão ou exclusão de substâncias nos padrões de potabilidade mundiais fundamenta-se, entre outros aspectos, no estudo de sua ocorrência -e potencial de ocorrência- nos mananciais de abastecimento (Cabral et al., 2012). Atualmente, são 496 substâncias autorizadas pala Agência Nacional de Vigilância Sanitária (ANVISA) (Agência Nacional de Vigilância Sanitária, 2020) para usos na agricultura, no domicílio como saneante, como preservante de madeira e para utilização em ambientes aquáticos ou não agrícolas, sendo que desses apenas 15 agrotóxicos constam no padrão brasileiro de potabilidade da água (Agência Nacional de Vigilância Sanitária, 2019b; Brasil, 2017).

Nesse sentido, este estudo tem por objetivo avaliar e discutir a dinâmica ambiental e a toxicidade dos IA de agrotóxicos de origem química que obtiveram seus registros aprovados pelo governo brasileiro no período de janeiro de 2018 a dezembro de 2019, bem como sugerir possíveis VMPs em água de consumo humano no Brasil, com base em dados obtidos em literatura.

\section{AVALIAÇÃO DOS AGROTÓXICOS AUTORIZADOS NO BRASIL}

\subsection{Evolução da autorização e proibição de ingredientes ativos no Brasil}

A Figura 1 apresenta o número de IA que foram autorizados ou proibidos pela ANVISA para uso no Brasil no período de 2012 a 2019, de acordo com as publicações no Diário Oficial da União. Cabe destacar que, nessa avaliação, não foram considerados os compostos classificados como adjuvantes, domissanitários, preservantes de madeira, hormônios de vegetais ou agentes microbiológicos. Em 2012, foram autorizados nove IA para uso no Brasil e, entre os anos de 2013 a 2017, o número de autorizações de IA variou de três a cinco por ano, sendo que nesse período nenhum IA foi proibido 
para uso no país. Em 2018, apenas um IA foi autorizado para uso no país e dois foram proibidos. Em 2019, diferentemente dos outros anos, 33 agrotóxicos foram proibidos para uso no Brasil, permanecendo a média de três IA autorizados ao ano (Agência Nacional de Vigilância Sanitária, 2019b, 2019c).

Especificamente no período de 2018 a 2019 (foco deste estudo), foram autorizados para uso no Brasil 15 novos compostos, sendo 11 deles agentes microbiológicos e excipientes e os demais agrotóxicos de origem química: Dinotefuran, Florpirauxifen-benzil, Fluopiram e Sulfoxaflor. Considerando o mesmo período, 35 compostos passaram a ser não autorizados pela ANVISA, tanto para a sua comercialização, quanto para a sua utilização, uma vez que não existiam produtos contendo esses IA com registro válido no Brasil (Agência Nacional de Vigilância Sanitária, 2019b, 2019c).

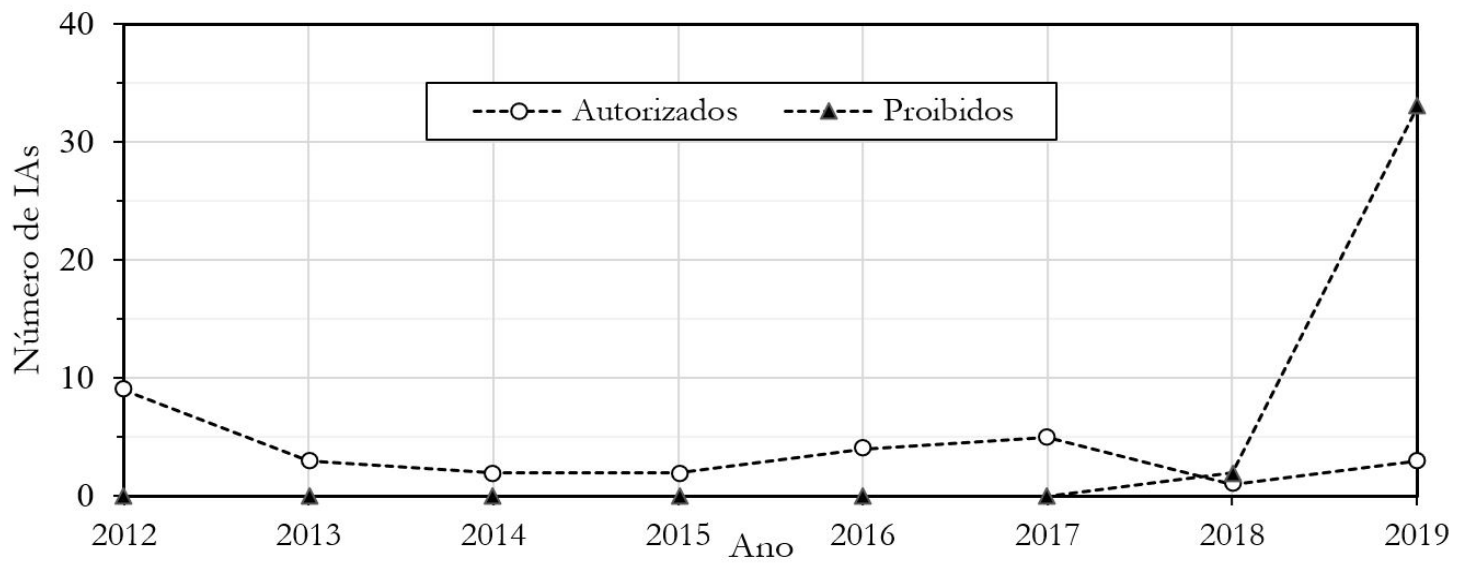

Figura 1 - Número de ingredientes ativos (IA) de agrotóxicos autorizados e proibidos no Brasil entre 2012 e 2019.

\subsection{Dinâmica ambiental dos agrotóxicos de origem química autorizados no Brasil entre 2018 e 2019}

Uma das etapas da AQRQ consiste na avaliação da exposição dos seres humanos, ou seja, da possibilidade daquele IA vir a ter contato com a população por diferentes meios. Assim, como o foco deste estudo é a exposição via ingestão de água, avaliou-se a possibilidade dos agrotóxicos em tela alcançarem os mananciais de águas superficiais e subterrâneas utilizando-se dos seguintes parâmetros comumente empregados na determinação da dinâmica ambiental de compostos lançados no ambiente (International Union of Pure and Applied Chemistry, 2019; Minnesota Department of Agriculture, 2012, 2013, 2018):

- $\quad$ Tempo de meia vida (DT50) em água e em solo: é o tempo necessário para que a concentração inicial do agrotóxico na água ou no solo decaia em $50 \%$ por meio de processos físico-químicos e biológicos de degradação. Para a avaliação da dinâmica ambiental dos agrotóxicos, foi utilizada a faixa de classificação recomendada pela IUPAC - International Union of Pure and Applied Chemistry (2019) para o DT50 em água: valores menores que um dia - rápida degradação; valores entre um e 14 dias - degradação moderadamente rápida; valores entre 14 e 30 dias - degradação lenta; e valores superiores a 30 dias - degradação estável. Dessa forma, para melhor representação na Tabela 1 foi utilizada a seguinte classificação: valores menores ou iguais a um dia - $\mathrm{DT}_{50}$ baixo; valores entre um e 14 dias - DT50 médio; e valores acima ou iguais a 14 dias $\mathrm{DT}_{50}$ alto. Para DT50 em solo foi adotada a seguinte classificação (New Zealand, 2018; National Pesticide Information Center, 2019; International Union of Pure and Applied Chemistry, 2019): valores menores ou iguais a 30 dias - DT $_{50}$ baixo; valores entre 30 e 100 dias - DT $_{50}$ médio; e valores acima ou iguais a 100 dias - DT 50 alto.

- Coeficiente de adsorção ao carbono orgânico do solo (Koc): reflete a tendência do agrotóxico se adsorver à fração orgânica do solo. A ATSDR - U.S. Agency for Toxic Substances and Disease Registry (Agency for Toxic Substances and Disease Registry, 1992), classifica essa tendência da seguinte forma: muito fraco (Koc $<10 \mathrm{~mL} / \mathrm{g})$, débil $(10 \mathrm{~mL} / \mathrm{g} \leq \mathrm{Koc}<100 \mathrm{~mL} / \mathrm{g})$, moderado $(100 \mathrm{~mL} / \mathrm{g} \leq$ Koc $<1000 \mathrm{~mL} / \mathrm{g})$, moderado a forte $(1000 \mathrm{~mL} / \mathrm{g} \leq \mathrm{Koc}<10000 \mathrm{~mL} / \mathrm{g})$, forte $(10000 \mathrm{~mL} / \mathrm{g} \leq \mathrm{Koc}<$ $100000 \mathrm{~mL} / \mathrm{g}$ ) e muito forte (Koc $\geq 100000 \mathrm{~mL} / \mathrm{g}$ ). Para melhor representação na Tabela 1, os 
resultados foram agrupados da seguinte forma: muito fraco e débil - baixo; moderado e moderado a forte - médio; e forte e muito forte - alto.

- Coeficiente de partição octanol/água (Kow): considera a relação das concentrações do agrotóxico em um solvente orgânico (octanol) e em água e, portanto, reflete a característica hidrofílica ou hidrofóbica da substância. Segundo Staudinger \& Roberts (1996), compostos que possuem valores de log Kow menor que um apresentam propriedades hidrofílicas (sendo considerado como baixo para efeitos deste estudo); compostos com log Kow maiores que três apresentam propriedades hidrofóbicas (sendo considerado como alto para efeitos deste estudo). Valores entre 1 e 3 foram considerados como médio.

- Volatilidade: a constante da lei de Henry $(\mathrm{KH})$ retrata a distribuição do composto entre as fases líquida e gasosa em função da temperatura, ou seja, o potencial de volatilização do agrotóxico a partir da água ou do solo. 0 mecanismo de volatilização é expressivo para compostos cujos valores da constante de Henry adimensional (KH') é superior a 10-4 (Stenstrom et al., 1989). Além disso, esse mecanismo pode ser influenciado pelo Kow, pois elevados valores desse coeficiente favorecem a retenção dos agrotóxicos nas frações sólidas e orgânicas da água e do solo em detrimento do processo de volatilização, sendo esse efeito observado por meio da relação entre o KH' e o Kow. Segundo Rogers (1996), valores da relação KH'/Kow menores que $10^{-9}$ indicam baixo potencial de volatilização do composto. Já valores maiores que $10^{-9}$ indicam alto potencial de volatilização.

- Solubilidade: considera a capacidade de dissolução de uma substância em um solvente, nesse caso, a água. Neste estudo, para avaliação da dinâmica ambiental dos agrotóxicos, a solubilidade dos compostos à $20^{\circ} \mathrm{C}$ foi classificada da seguinte forma (International Union of Pure and Applied Chemistry, 2019): valores menores ou iguais a $50 \mathrm{mg} / \mathrm{L}$ - baixa; valores entre 50 e $500 \mathrm{mg} / \mathrm{L}$ moderada; e valores superiores a $500 \mathrm{mg} / \mathrm{L}$ - alta solubilidade.

A Tabela 1 traz uma compilação desses parâmetros para os agrotóxicos selecionados, apresentando o potencial de contaminação das águas superficiais e subterrâneas por esses compostos. 0 potencial de contaminação das águas superficiais e subterrâneas foi avaliado segundo os índices GOSS (Goss, 1992) e GUS, respectivamente (Gustafson, 1989). Esses índices consideram as características físico-químicas dos compostos (Koc, solubilidade em água e DT50 no solo) para classificação do potencial de contaminação das referidas matrizes ambientais. Salienta-se ainda que os dados utilizados para a confecção da Tabela 1 foram obtidos da International Union of Pure and Applied Chemistry (2019) e do Departamento de Agricultura de Minnesota, EUA (Minnesota Department of Agriculture, 2012, 2013, 2018).

Com base na Tabela 1, de início é possível notar a elevada persistência ambiental dos agrotóxicos autorizados no Brasil entre 2018 e 2019, notadamente do Fluopiram (vide intensidade dos DT50). A título de exemplo, Dinotefuran, Fluopiram e Sulfoxaflor apresentaram $\mathrm{DT}_{50}$ em água maiores que 20 dias. No caso do Dinotefuran, observa-se ainda elevada mobilidade ambiental (índices GUS e GOSS altos; baixos valores de Koc e Kow, e elevada solubilidade em água) o que do ponto de vista da AQRQ pode indicar a possibilidade de exposição de seres humanos a elevados níveis do agrotóxico via consumo de água, caso haja contaminação do ambiente. Já a mobilidade do Fluopiram encontra-se em um nível intermediário, pois apesar da baixa solubilidade em água e do elevado Kow, seu valor intermediário de Koc (aliado ao elevado $\mathrm{DT}_{50}$ no solo) e seus elevados índices GOSS e GUS denotam possibilidade de contaminação das águas superficiais e subterrâneas. Para o Florpirauxifen-benzil, parâmetros como solubilidade em água, Koc e Kow sugerem elevada capacidade de retenção em solo e sedimentos de ambientes aquáticos com alto teor de matéria orgânica, o que em outras palavras denotam baixa mobilidade ambiental e menor possibilidade de exposição de seres humanos via consumo de água. No entanto, cabe destacar que tanto Florpirauxifen-benzil quanto Fluopiram apresentam elevados $\mathrm{DT}_{50}$ no solo, podendo os seres humanos estarem sujeitos a outras vias de exposição (Ex.: ingestão de alimentos contaminados). Nos tópicos a seguir a dinâmica ambiental de cada composto é apresentada em maiores detalhes. 
Tabela 1 - Intensidade dos parâmetros utilizados para a avaliação da dinâmica ambiental dos agrotóxicos de origem química autorizados no Brasil entre 2018 e 2019

\begin{tabular}{|c|c|c|c|c|c|c|c|c|c|}
\hline 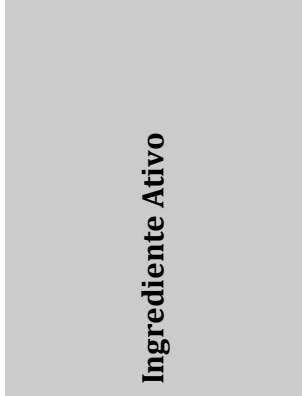 & 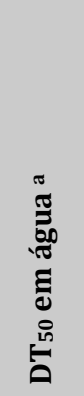 & $\begin{array}{l}0 \\
\stackrel{0}{0} \\
\dot{0} \\
0 \\
0 \\
0 \\
0 \\
\dot{n} \\
\dot{n}\end{array}$ & 峁 & 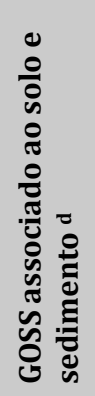 & 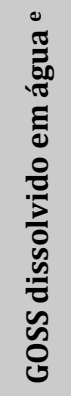 & $\ddot{y}$ & $\sum_{2}^{\infty}$ & 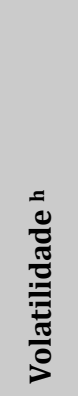 & 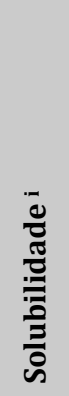 \\
\hline Dinotefuran & +++ & ++ & +++ & ++ & +++ & + & + & + & +++ \\
\hline Florpirauxifen-benzil & ++ & +++ & + & +++ & ++ & +++ & +++ & +++ & + \\
\hline Fluopiram & +++ & +++ & +++ & ++ & +++ & ++ & +++ & + & + \\
\hline Sulfoxaflor & +++ & + & + & + & ++ & + & + & + & +++ \\
\hline
\end{tabular}

+++ Alto; ++ Médio; + Baixo. DT50: tempo de meia vida. GOSS: potencial de contaminação das águas superficiais (Goss, 1992). GUS $=\log \left(\mathrm{DT}_{50}\right.$ solo $) \times(4-\log (\mathrm{Koc}))$, indica o potencial de contaminação das águas subterrâneas (Gustafson, 1989). Koc: coeficiente de adsorção ao solo. Kow: coeficiente de partição octanol/água. a: + (DT50 $\leq 1 \mathrm{~d}) ;++\left(1 \mathrm{~d}<\mathrm{DT}_{50}<14 \mathrm{~d}\right) ;+++\left(\mathrm{DT}_{50}\right.$ $\geq 14 \mathrm{~d}) \mathrm{b}:+\left(\mathrm{DT}_{50} \leq 30 \mathrm{~d}\right) ;++\left(30 \mathrm{~d}<\mathrm{DT}_{50}<100 \mathrm{~d}\right) ;+++\left(\mathrm{DT}_{50} \geq 100 \mathrm{~d}\right)$. c: + (GUS $\left.\leq 1,8\right) ;++(1,8<\mathrm{GUS}<2,8) ;+++(\mathrm{GUS} \geq 2,8)$ $\mathrm{d}:+\left(\mathrm{DT}_{50}\right.$ solo $\left.\leq 1 \mathrm{~d}\right)$ ou $\left(\mathrm{DT}_{50}\right.$ solo $\leq 2 \mathrm{~d}$ e Koc $\left.\leq 500 \mathrm{~mL} / \mathrm{g}\right)$ ou $\left(\mathrm{DT}_{50}\right.$ solo $\leq 4 \mathrm{~d}$ e Koc $\leq 900 \mathrm{~mL} / \mathrm{g}$ e Solubilidade $\left.\geq 0,5 \mathrm{mg} / \mathrm{L}\right)$ ou $\left(\mathrm{DT}_{50}\right.$ solo $\leq 40 \mathrm{~d}$ e Koc $\leq 500 \mathrm{~mL} / \mathrm{g}$ e Solubilidade $\geq 0,5 \mathrm{mg} / \mathrm{L}$ ) ou (DT 50 solo $\leq 40 \mathrm{~d}$ e Koc $\leq 900 \mathrm{~mL} / \mathrm{g}$ e Solubilidade $\geq 2$ $\mathrm{mg} / \mathrm{L}) ;++$ os que não se enquadraram em + ou +++; +++ (DT50 solo $\geq 40 \mathrm{~d}$ e Koc $\geq 1000 \mathrm{~mL} / \mathrm{g}$ ) ou (DT50 solo $\geq 40 \mathrm{~d}$ e Koc $\geq$ $500 \mathrm{~mL} / \mathrm{g}$ e Solubilidade $\leq 0,5 \mathrm{mg} / \mathrm{L})$. e: $+($ Koc $\geq 100000 \mathrm{~mL} / \mathrm{g})$ ou (Koc $\geq 1000 \mathrm{~mL} / \mathrm{g}$ e DT 50 solo $\leq 1 \mathrm{~d}$ ) ou (Solubilidade $<$ $0,5 \mathrm{mg} / \mathrm{L}$ e $\mathrm{DT}_{50}$ solo $\left.<35 \mathrm{~d}\right) ;++$ os que não se enquadraram em + ou +++; +++ (Solubilidade $\geq 1 \mathrm{mg} / \mathrm{L} \mathrm{e} \mathrm{DT} 50$ solo $>35 \mathrm{~d}$ e Koc $<100000 \mathrm{~mL} / \mathrm{g}$ ) ou (Solubilidade $\geq 10 \mathrm{mg} / \mathrm{L}$ e Solubilidade $<100 \mathrm{mg} / \mathrm{L}$ e Koc $\leq 700 \mathrm{~mL} / \mathrm{g})$. f: $+(\mathrm{Koc}<100 \mathrm{~mL} / \mathrm{g}) ;++(100$ $\mathrm{mL} / \mathrm{g} \leq \mathrm{Koc}<10000 \mathrm{~mL} / \mathrm{g}) ;+++(\mathrm{Koc} \geq 10000 \mathrm{~mL} / \mathrm{g}) \cdot \mathrm{g}:+(\log \mathrm{Kow}<1) ;++(1<\log \operatorname{Kow}<3) ;+++(\log \mathrm{Kow}>3)$. h: $+\left(\mathrm{KH}^{\prime}<\right.$ $10^{-4}$ e $\left.\mathrm{KH}^{\prime} / \mathrm{Kow}<10^{-9}\right)$; ++ $\left(\mathrm{KH}^{\prime}>10^{-4}\right.$ e KH'/Kow < 10-9); +++ $\left(\mathrm{KH}^{\prime}>10^{-4} \mathrm{e} \mathrm{KH}^{\prime} / \mathrm{Kow}>10^{-9}\right)$. i: + (Solubilidade $\left.\leq 50 \mathrm{mg} / \mathrm{L}\right)$; $++(50 \mathrm{mg} / \mathrm{L}<$ Solubilidade $<500 \mathrm{mg} / \mathrm{L}) ;+++($ Solubilidade $\geq 500 \mathrm{mg} / \mathrm{L})$

\subsection{Efeitos à saúde pela exposição crônica aos agrotóxicos de origem química autorizados no Brasil entre 2018 e 2019 e possíveis valores normativos para a concentração em água de consumo humano}

Na AQRQ, o nível de risco associado a uma substância química é determinado pelo conjunto do grau de exposição e da toxicidade. Para a determinação da toxicidade, os riscos toleráveis são tipicamente avaliados com base nos efeitos críticos observados em estudos crônicos de dose-resposta com animais ou humanos (epidemiológicos), em geral considerando-se as doses mais altas que não causaram efeitos adversos (NOAEL - No Observed Adverse Effect Level) e o efeito observado. A partir desses dados, são aplicados fatores de segurança (ou de incerteza - FI) para extrapolação das doses para humanos, obtendo-se a ingestão diária aceitável (IDA) por seres humanos. No estabelecimento de valores-guia ou valores máximos permitidos (VMP) para compostos químicos em água de consumo humano, atribui-se uma proporção da IDA para a via de exposição pela ingestão de água (fator de alocação - FA), sendo a proporção restante atribuída a outras vias de exposição (Ex.: ingestão de alimentos). 0 valor numérico do VMP reflete que ao longo da vida (exposição crônica), caso haja a ingestão diária de dois litros de água por uma pessoa de $60 \mathrm{~kg}$, haverá uma baixa probabilidade de ocorrência de danos à saúde. Para compostos carcinogênicos, os valores-guia ou VMP são tipicamente baseados em extrapolações dos estudos dose-resposta, em que são estabelecidas as doses correspondentes aos riscos adicionais de câncer na população.

A Tabela 2 apresenta uma compilação dos estudos toxicológicos desenvolvidos com os agrotóxicos de origem química autorizados no Brasil entre 2018 e 2019, informações essas obtidas em agências/entidades renomadas como: as monografias da ANVISA e da EFSA - European Food Safety Authority (Agência Nacional de Vigilância Sanitária, 2019b; European Food Safety Authority, 2019), os perfis toxicológicos da Agency for Toxic Substances and Disease Registry (2019), a diretriz de qualidade da água para consumo humano da Organização Mundial da Saúde (OMS) (World Health Organization, 2017) e as diretrizes/normas de qualidade da água para consumo humano dos EUA, Nova Zelândia, Austrália e Canadá (United States Environmental Protection Agency, 2018a; New 
Zealand, 2018; National Health and Medical Research Council, 2018; Health Canada, 2017). Vale notar que, a despeito das referências aos estudos toxicológicos, nenhum dos quatro IA possuem valoresnormativos para águas de consumo humano instituídos pelas entidades/agências dos diferentes países consultados (Brasil, 2017; World Health Organization, 2017; Health Canada, 2017; National Health and Medical Research Council, 2018; United States Environmental Protection Agency, 2018a; New Zealand, 2018).

Conforme mostra a Tabela 2, dentre os agrotóxicos autorizados para uso no Brasil nos anos 2018 e 2019, o Sulfoxaflor e o Fluopiram apresentam maior toxicidade (menor IDA), enquanto que o Florpirauxifen-benzil apresentou menor toxicidade (maior IDA). Nesse viés, os valores-normativos calculados mais restritivos foram para o Sulfoxaflor (62 a $308 \mu \mathrm{g} / \mathrm{L}$ ) e Fluopiram (72 $\mu \mathrm{g} / \mathrm{L}$ ), e os menos restritivos para o Florpirauxifen-benzil (3.000 a $14.400 \mu \mathrm{g} / \mathrm{L}$ ) (Tabela 2). Ainda que o Sulfoxaflor apresente elevada toxicidade (Tabela 2) e elevada persistência ambiental (DT50 na água na Tabela 1), vale lembrar que o Sulfoxaflor apresenta baixa mobilidade ambiental (demais parâmetros da Tabela 1) e, portanto, baixo potencial de contaminação dos mananciais de abastecimento de água. Já o Dinotefuran e o Fluopiram são agrotóxicos que também apresentam toxicidade alta (Tabela 2), no entanto apresentam considerável persistência/mobilidade ambiental (Tabela 1), sugerindo um maior risco do ponto de vista da exposição de seres humanos via consumo de água. No caso do Florpirauxifen-benzil, a sua baixa toxicidade (Tabela 2) e baixa mobilidade ambiental (Tabela 1) sugerem um menor risco da exposição de seres humanos via consumo de água.

No próximo tópico os compostos serão discutidos separadamente de forma a contemplar suas particularidades.

Tabela 2- Valores calculados para a ingestão diária aceitável (IDA) e para a concentração correspondente em água de consumo humano (possível valor-normativo).

\begin{tabular}{|c|c|c|c|c|c|c|c|c|}
\hline 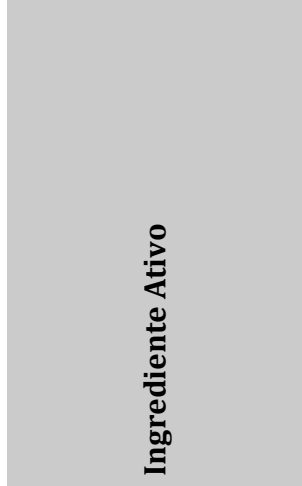 & 丞 & 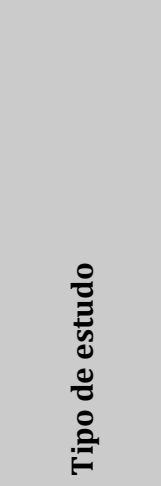 & 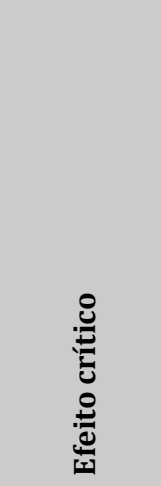 & 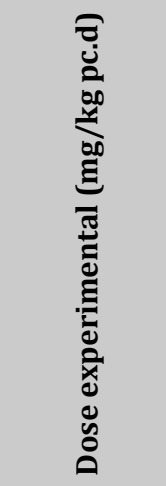 & 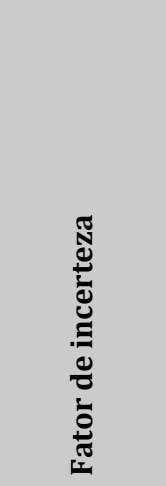 & 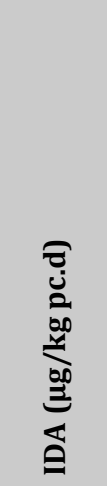 & 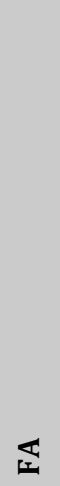 & 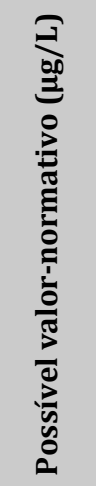 \\
\hline & $\begin{array}{c}\text { Agência Nacional de } \\
\text { Vigilância Sanitária } \\
(2019 b)\end{array}$ & $\begin{array}{c}\text { Não } \\
\text { informado. }\end{array}$ & $\begin{array}{c}\text { Não } \\
\text { informado. }\end{array}$ & $\begin{array}{c}\text { Não } \\
\text { informado. }\end{array}$ & $\begin{array}{c}\text { Não } \\
\text { informado. }\end{array}$ & 22,0 & 0,2 & 132 \\
\hline Dinotefuran & \begin{tabular}{|c|} 
United States \\
Environmental \\
Protection Agency \\
(2004) European \\
Food Safety Authority \\
(2014a) Australian \\
Pesticides and \\
Veterinary Medicines \\
Authority (2015a) \\
Joint FAO/WHO \\
Meeting on Pesticide \\
Residues (2012) \\
\end{tabular} & $\begin{array}{c}\text { Estudo } \\
\text { crônico } \\
\text { com cães. }\end{array}$ & $\begin{array}{c}\text { Redução } \\
\text { do peso e } \\
\text { consumo } \\
\text { de } \\
\text { alimentos. }\end{array}$ & $\begin{array}{c}22,0 \\
\text { (NOAEL) }\end{array}$ & 100 & 22,0 & 0,2 & 132 \\
\hline \multirow[b]{2}{*}{ Florpirauxifen-benzil } & $\begin{array}{c}\text { Agência Nacional de } \\
\text { Vigilância Sanitária } \\
(2019 b)\end{array}$ & $\begin{array}{c}\text { Não } \\
\text { informado. }\end{array}$ & $\begin{array}{c}\text { Não } \\
\text { informado. }\end{array}$ & $\begin{array}{c}\text { Não } \\
\text { informado. }\end{array}$ & $\begin{array}{c}\text { Não } \\
\text { informado. }\end{array}$ & $2.400,0$ & 0,2 & 14.400 \\
\hline & $\begin{array}{l}\text { European Food Safety } \\
\text { Authority (2018) }\end{array}$ & $\begin{array}{c}\text { Estudo } \\
\text { crônico } \\
\text { com ratos. }\end{array}$ & $\begin{array}{l}\text { Tumores } \\
\text { na } \\
\text { glândula } \\
\text { mamária }\end{array}$ & $\begin{array}{c}50,0 \\
\text { (NOAEL) }\end{array}$ & 100 & 500,0 & 0,2 & 3.000 \\
\hline
\end{tabular}


Tabela 2- Continuação...

\begin{tabular}{|c|c|c|c|c|c|c|c|c|}
\hline 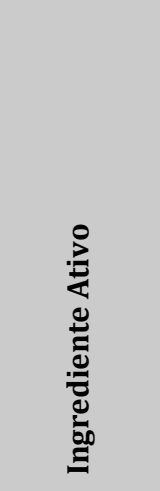 & 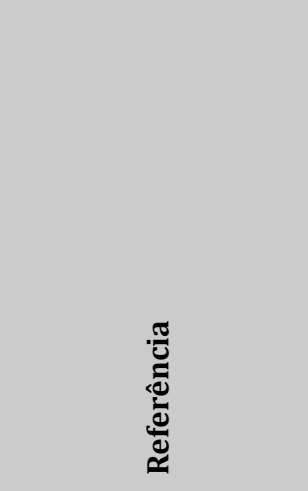 & 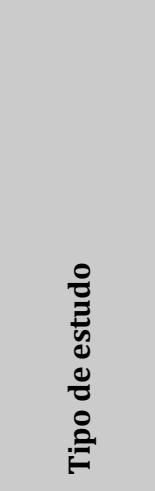 & $\begin{array}{l}: \\
: \\
:\end{array}$ & 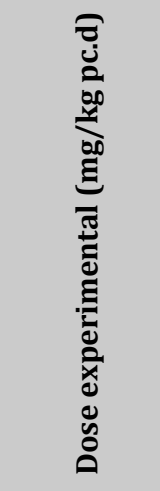 & 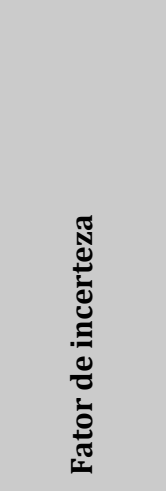 & 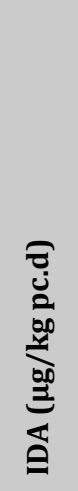 & 厌 & 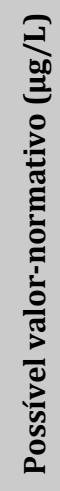 \\
\hline & $\begin{array}{l}\text { Agência Nacional de } \\
\text { Vigilância Sanitária } \\
(2019 \mathrm{~b})\end{array}$ & $\begin{array}{c}\text { Não } \\
\text { informado. }\end{array}$ & $\begin{array}{c}\text { Não } \\
\text { informado. }\end{array}$ & $\begin{array}{c}\text { Não } \\
\text { informado. }\end{array}$ & $\begin{array}{c}\text { Não } \\
\text { informado. }\end{array}$ & 12,0 & 0,2 & 72 \\
\hline Fluopiram & $\begin{array}{l}\text { Australian Pesticides } \\
\text { and Veterinary } \\
\text { Medicines Authority } \\
\text { (2015b) United States } \\
\text { Environmental } \\
\text { Protection Agency } \\
\text { (2019) European } \\
\text { Food Safety Authority } \\
\text { (2013) Joint } \\
\text { FAO/WHO Meeting on } \\
\text { Pesticide Residues } \\
\text { (2010) }\end{array}$ & \begin{tabular}{|c|} 
Estudo \\
crônico \\
com ratos.
\end{tabular} & \begin{tabular}{|} 
Efeitos no \\
fígado, \\
rim, \\
tireoide e \\
olhos.
\end{tabular} & $\begin{array}{c}1,2 \\
\text { (NOAEL) }\end{array}$ & 100 & 12,0 & 0,2 & 72 \\
\hline \multirow{3}{*}{ Sulfoxaflor } & $\begin{array}{l}\text { Agência Nacional de } \\
\text { Vigilância Sanitária } \\
\qquad(2019 b)\end{array}$ & $\begin{array}{c}\text { Não } \\
\text { informado. }\end{array}$ & $\begin{array}{c}\text { Não } \\
\text { informado. }\end{array}$ & $\begin{array}{c}\text { Não } \\
\text { informado. }\end{array}$ & $\begin{array}{c}\text { Não } \\
\text { informado. }\end{array}$ & 10,4 & 0,2 & 62 \\
\hline & $\begin{array}{l}\text { Australian Pesticides } \\
\text { and Veterinary } \\
\text { Medicines Authority } \\
\text { (2013) European } \\
\text { Food Safety Authority } \\
\text { (2014b) } \\
\end{array}$ & \begin{tabular}{|c|} 
Estudo \\
crônico \\
com ratos
\end{tabular} & $\begin{array}{l}\text { Efeitos no } \\
\text { fígado. }\end{array}$ & $\begin{array}{c}4,24 \\
\text { (NOAEL) }\end{array}$ & 100 & 42,4 & 0,2 & 254 \\
\hline & \begin{tabular}{|c} 
United States \\
Environmental \\
Protection Agency \\
(2013) Joint \\
FAO/WHO Meeting on \\
Pesticide Residues \\
(2011)
\end{tabular} & \begin{tabular}{|c|} 
Estudo \\
crônico \\
com ratos.
\end{tabular} & $\begin{array}{l}\text { Efeitos no } \\
\text { fígado. }\end{array}$ & $\begin{array}{c}5,13 \\
\text { (NOAEL) }\end{array}$ & 100 & 51,3 & 0,2 & 308 \\
\hline
\end{tabular}

IDA: Ingestão diária aceitável; FA: fator de alocação de 20\% da IDA para a exposição via consumo de água (World Health Organization, 2017); NOAEL: dose para efeito adverso não observado: nível de exposição mais elevado para o qual não se observam efeitos adversos;

Para o cálculo da IDA, tem-se: $\operatorname{IDA}(\mu \mathrm{g} / \mathrm{L})=\frac{\text { Dose experimental }(\mu \mathrm{g} / \mathrm{kg} / \mathrm{d})}{\text { Fator de incerteza }}$.

O cálculo do valor-guia foi feito com base na equação: Valor guia $(\mu \mathrm{g} / \mathrm{L})=\frac{\mathrm{IDA}(\mu \mathrm{g} / \mathrm{kg} / \mathrm{d}) \times \mathrm{PC}(\mathrm{kg}) \times \mathrm{FA}}{\mathrm{V}(\mathrm{L} / \mathrm{d})}$, sendo PC: peso corpóreo médio de $60 \mathrm{~kg}$ e V: consumo de água diário de 2,0L (World Health Organization, 2017).

\section{DINOTEFURAN}

0 inseticida Dinotefuran foi autorizado para uso no Brasil em agosto de 2019, podendo ser aplicado em cultivos de arroz, aveia, batata, café, cana-de-açúcar, centeio, cevada, citros, feijão, milheto, milho, pastagem, soja, tomate, trigo e triticale (Agência Nacional de Vigilância Sanitária, 
2019b). Nesse sentido, vale notar que esse IA é autorizado para uso em importantes culturas no país, sendo a soja e o milho as duas culturas de maior relevância em área plantada no Brasil no ano 2018 (aproximadamente 34,8 e 16,5 milhões de hectares plantados, respectivamente) (Instituto Brasileiro de Geografia e Estatística, 2019). A relevância desses cultivos também é explicitada quando se avalia o valor da produção: somente em 2018, a soja movimentou cerca de 127,5 bilhões de reais e o milho 37,6 bilhões de reais (Instituto Brasileiro de Geografia e Estatística, 2019).

$0 \mathrm{DT}_{50}$ em água e no solo para esse IA é de, respectivamente, 80,8 dias e 82 dias, assim sendo, 0 IA é considerado persistente em ambas as matrizes (United States Environmental Protection Agency, 2004; International Union of Pure and Applied Chemistry, 2019). Esse IA também apresenta alto potencial de contaminação das águas subterrâneas e superficiais (Gustafson, 1989; Goss, 1992). Possui baixo Koc, indicando baixa capacidade de adsorção ao solo e aos sedimentos em ambientes aquáticos, o que reforça a sua elevada mobilidade ambiental. 0 log Kow para o Dinotefuran é menor que um, indicando ser um composto hidrofílico (Staudinger \& Roberts, 1996), o que pode ser confirmado por sua elevada solubilidade em água. $\mathrm{O}$ valor de $\mathrm{KH}^{\prime}$ inferior a $10^{-4}$ e a relação $\mathrm{KH}^{\prime} /$ Kow menor que $10^{-9}$, classificam o inseticida como pouco volátil (Stenstrom etal., 1989; Rogers, 1996). Não foram encontrados dados sobre a ocorrência do Dinotefuran em águas superficiais ou subterrâneas do Brasil.

O Dinotefuran não apresenta classificação carcinogênica pela Agência Internacional de Pesquisa em Câncer, International Agency for Research on Cancer (2019), entretanto a Agência de Proteção Ambiental dos Estados Unidos (United States Environmental Protection Agency, 2018b) o classifica como não carcinogênico para seres humanos, devido à falta de evidência de carcinogenicidade e mutagenicidade relacionada à exposição de camundongos e ratos a esse composto (United States Environmental Protection Agency, 2004). As agências Agência Nacional de Vigilância Sanitária (2019b), European Food Safety Authority (2014a) e APVMA (Australian Pesticides and Veterinary Medicines Authority) (2015a) determinaram $22 \mu \mathrm{g} / \mathrm{kg}$ pc.d como valor de IDA, enquanto United States Environmental Protection Agency (2004) e Joint FAO/WHO Meeting on Pesticide Residues (2012) recomendaram $20 \mu \mathrm{g} / \mathrm{kg}$ pc.d, cujo valor foi obtido com base no mesmo NOAEL adotado pelas demais agências internacionais.

Por meio da IDA reportada pela Agência Nacional de Vigilância Sanitária (2019b) e da dose experimental de $22 \mathrm{mg} / \mathrm{kg}$ pc.d considerada pelas demais agências (United States Environmental Protection Agency, 2004; European Food Safety Authority, 2014a; Joint FAO/WHO Meeting on Pesticide Residues, 2012; Australian Pesticides and Veterinary Medicines Authority, 2015a), um valornormativo sugerido para concentração máxima de Dinotefuran em água potável seria de $132 \mu \mathrm{g} / \mathrm{L}$ (Tabela 2). Por meio de estudo toxicológico com cães, observou-se a redução do ganho de peso e do consumo de alimentos como resposta à ingestão crônica desse IA (United States Environmental Protection Agency, 2004; European Food Safety Authority, 2014a; Joint FAO/WHO Meeting on Pesticide Residues, 2012; Australian Pesticides and Veterinary Medicines Authority, 2015a).

Por fim, nota-se que o Dinotefuran é um composto que tem uso autorizado em culturas de destaque no Brasil, possuindo uma dinâmica ambiental favorável à contaminação dos mananciais de abastecimento de água. Por outro lado, o efeito observado nos estudos de toxicidade não se apresenta de elevada severidade, resultando em redução do ganho de peso e do consumo de alimentos. Todavia, entre as agências consultadas, há um consenso no valor de IDA, resultando em um possível valor normativo de $132 \mu \mathrm{g} / \mathrm{L}$. Assim, recomenda-se o acompanhamento da comercialização de tal composto, assim como o seu monitoramento em mananciais de abastecimento de água, a fim de se verificar a necessidade de regulamentação desse inseticida na portaria de potabilidade da água de consumo humano do Brasil.

\section{FLORPIRAUXIFEN-BENZIL}

O herbicida Florpirauxifen-benzil teve seu registro autorizado para comercialização e utilização no Brasil em maio de 2019, sendo autorizado pela ANVISA para aplicação em pós-emergência nos cultivos de arroz, café, citros, fumo e couve (Agência Nacional de Vigilância Sanitária, 2019b). Destacase que a plantação de arroz no país ocupou cerca de 1,9 milhões de hectares em 2018 e o valor da produção foi de aproximadamente 8,6 bilhões de reais (Instituto Brasileiro de Geografia e Estatística, 2019).

Considerando sua dinâmica ambiental (Tabela 1), esse agrotóxico apresenta DT 50 em água médio, em torno de 3,3 dias, e alto DT $_{50}$ em solo, 150 dias, degradando moderamente rápido em água e lentamente no solo (International Union of Pure and Applied Chemistry, 2019). Possui baixo potencial de contaminação das águas subterrâneas e médio potencial de contaminação das águas superficiais 
(Gustafson, 1989; Goss, 1992). 0 Koc é da ordem de $32308 \mathrm{~mL} / \mathrm{g}$, indicando ter alta capacidade de adsorção ao conteúdo orgânico do solo e, portanto, baixa mobilidade nessa matriz (International Union of Pure and Applied Chemistry, 2019; Agency for Toxic Substances and Disease Registry, 1992). 0 log Kow para esse composto é 5,46, considerado alto, típico de um composto hidrofóbico (International Union of Pure and Applied Chemistry, 2019; Staudinger \& Roberts, 1996), dado confirmado por sua baixa solubilidade em água. A relação KH'/Kow é maior que $10^{-9}$ e o valor de $\mathrm{KH}^{\prime}$ é superior a $10^{-4}$, sendo classificado como alto, o que indica o alto potencial de volatilidade do composto (Rogers, 1996; Stenstrom et al., 1989). Utilizando a metodologia MEDRICE (metodologia desenvolvida pela União Europeia (European Commission, 2003) a fim de predizer concentrações de agrotóxicos em águas superficiais e sedimentos em cultivos de arroz), a European Food Safety Authority (2018) constataram que a concentração do Florpirauxifen-benzil em águas subterrâneas não ultrapassaria $0,1 \mu \mathrm{g} / \mathrm{L}$. Contudo, não foram encontrados na literatura consultada estudos que reportassem a ocorrência desse composto em mananciais de abastecimento.

O Florpirauxifen-benzil não apresenta classificação carcinogênica pela International Agency for Research on Cancer (2019), todavia a United States Environmental Protection Agency (2018b) o classifica como não carcinogênico para humanos. A IDA estabelecida pela Agência Nacional de Vigilância Sanitária (2019b) para esse composto é de $24.000 \mu \mathrm{g} / \mathrm{kg}$ pc.d, valor esse superior à IDA que foi determinado por European Food Safety Authority (2018), que é de $500 \mu \mathrm{g} / \mathrm{kg}$ pc.d (Tabela 2). A Australian Pesticides and Veterinary Medicines Authority (2018) não estabelece um valor para a IDA por considerar baixo o potencial de ocorrência de efeitos toxicológicos resultantes da ingestão crônica desse IA e pela falta de evidências quanto ao seu potencial carcinogênico, neurotóxico e imunotóxico.

Diante da IDA preconizada pela Agência Nacional de Vigilância Sanitária (2019b), tem-se um possível valor-normativo de $14.400 \mu \mathrm{g} / \mathrm{L}$ para o Florpirauxifen-benzil em água potável (Tabela 2). Já considerando uma dose experimental de $50 \mathrm{mg} / \mathrm{kg}$ pc.d (European Food Safety Authority, 2018), um possível valor-normativo para água potável de $3.000 \mu \mathrm{g} / \mathrm{L}$ poderia ser adotado (Tabela 2). Segundo os estudos toxicológicos de curto prazo com ratos, os efeitos da ingestão desse IA incluem mineralização dos túbulos renais, alteração do pH da urina e redução do ganho de peso, chegando a tumores nas glândulas mamárias de ratos, observados em estudo crônico pelo qual se baseia a IDA preconizada por European Food Safety Authority (2018). Apesar disso, a EFSA não classifica o Florpirauxifen-benzil como carcinogênico, devido às incertezas identificadas no estudo toxicológico em questão, não sendo assegurado, até então, que a ocorrência de tumores nos animais se deu como resposta da ingestão do IA (European Food Safety Authority, 2018).

Dessa forma, considerando a forte afinidade do Florpirauxifen-benzil pelo carbono orgânico do solo ou dos sedimentos dos ambientes aquáticos (Koc $32308 \mathrm{~mL} / \mathrm{g}$ ), considerando que o composto possui um $\mathrm{DT}_{50}$ no solo de 150 d e elevada volatilidade, de fato, é possível dizer que esse agrotóxico possui baixa probabilidade de ocorrência em mananciais superficiais e subterrâneos. Mesmo se considerada uma forma de aplicação do IA que o leve diretamente para a água (Ex.: pulverização acidental diretamente sobre um corpo hídrico), seu DT 50 nessa matriz é curto (3,3 d), confirmando a baixa probabilidade de ocorrência na água. Ao verificar sua toxicidade com base na IDA mais restritiva, chega-se a um possível VMP de $3 \mathrm{mg} / \mathrm{L}$, o que é extremamente elevado e improvável de ocorrer em mananciais de abastecimento de água. Assim, com base na sua toxicidade e dinâmica ambiental, caso não se tenham novas evidências, a princípio não se deve estabelecer um nível de alerta para a ocorrência do Florpirauxifen-benzil em mananciais de abastecimento de água.

\section{FLUOPIRAM}

O fungicida Fluopiram foi autorizado para uso no Brasil em agosto de 2019, podendo ser aplicado nas seguintes culturas: algodão, batata, café, cana-de-açúcar, feijão, milho e soja (Agência Nacional de Vigilância Sanitária, 2019b). Assim como o Dinotefuran, esse agrotóxico possui autorização para aplicação nos principais cultivos brasileiros, sendo eles: a soja e o milho.

Conforme explicitado na Tabela 1, o Fluopiram apresenta DT50 na água e solo de 20,5 dias e 309 dias, respectivamente, o que é considerado como alto e, portanto, mostra que o composto se degrada lentamente tanto no solo quanto na água (International Union of Pure and Applied Chemistry, 2019). Avaliando-se os índices GOSS e GUS, o Fluopiram possui alto potencial de contaminação das águas superficiais e subterrâneas e médio potencial de associação ao solo e ao sedimento (Goss, 1992; Gustafson, 1989). 0 Koc é classificado como médio, sendo moderadamente adsorvido ao solo (Agency for Toxic Substances and Disease Registry, 1992). Em contrapartida, o valor do log Kow é maior que três, indicando ser um composto hidrofóbico (Staudinger \& Roberts, 1996). O composto ainda possui baixa solubilidade em água 
(International Union of Pure and Applied Chemistry, 2019), relação KH'/Kow inferior ao valor de $10^{-9}$ e KH' menor que $10^{-4}$, indicando baixa volatilidade (Rogers, 1996; Stenstrom et al., 1989). Portanto, devido à alta persistência no solo e mobilidade ambiental moderada do Fluopiram, esse em períodos muito longos pode atingir as águas subterrâneas mais vulneráveis, contudo, pressupõe-se que em concentrações muito baixas e abaixo dos níveis considerados nocivos para os organismos aquáticos (Australian Pesticides and Veterinary Medicines Authority, 2015b). Nas fontes consultadas não foram encontrados dados de ocorrência do Fluopiram em águas superficiais ou subterrâneas do Brasil.

O Fluopiram não é classificado como carcinogênico pela International Agency for Research on Cancer (2019) ou pela United States Environmental Protection Agency (2018b). As agências Agência Nacional de Vigilância Sanitária (2019b), United States Environmental Protection Agency (2019), European Food Safety Authority (2013) e Australian Pesticides and Veterinary Medicines Authority (2015b) estabeleceram um valor de IDA de $12 \mu \mathrm{g} / \mathrm{kg}$ pc.d (Tabela 2). Já a Joint FAO/WHO Meeting on Pesticide Residues (2010) propôs um intervalo de IDA entre 0 e $10 \mu \mathrm{g} / \mathrm{kg}$ pc.d, sendo esse um valor obtido a partir do mesmo estudo toxicológico que embasa a IDA das demais agências.

A partir do NOAEL de 1,2 mg/kg pc.d relativo à um estudo crônico com ratos do qual baseiam-se as IDA de todas as agências internacionais consultadas (Joint FAO/WHO Meeting on Pesticide Residues, 2010; United States Environmental Protection Agency, 2019; European Food Safety Authority, 2013; Australian Pesticides and Veterinary Medicines Authority, 2015b), tem-se um possível valor-normativo de $72 \mu \mathrm{g} / \mathrm{L}$ na água potável (Tabela 2), o mesmo obtido por meio da IDA reportada pela Agência Nacional de Vigilância Sanitária (2019b), provavelmente advindo do mesmo estudo utilizado pelas demais agências. Dentre os possíveis efeitos resultantes da ingestão crônica desse fungicida, estão a perda de peso corpóreo, o aumento do peso dos rins, a hipertrofia hepatocelular, a eosinofilia e a hiperplasia folicular na tireoide (Joint FAO/WHO Meeting on Pesticide Residues, 2010; Australian Pesticides and Veterinary Medicines Authority, 2015b). Destacam-se ainda a ocorrência de nefropatia e adenomas e carcinomas hepatocelulares e foliculares em ratos, sendo que a nefropatia não foi considerada como um efeito relevante a humanos. Os tumores foliculares também não foram considerados relevantes à humanos devido ao seu modo de ação (Australian Pesticides and Veterinary Medicines Authority, 2015b). No caso dos tumores hepatocelulares, considerou-se que a relevância de sua ocorrência em humanos não pôde ser descartada, sendo necessários estudos posteriores (Australian Pesticides and Veterinary Medicines Authority, 2015b).

Portanto, por possuir uma dinâmica ambiental favorável à contaminação das águas, ter uma elevada toxicidade, haver possibilidade de tumores hepatocelulares e considerando que pode ser utilizado em culturas de destaque no Brasil, recomenda-se o monitoramento do composto em mananciais de abastecimento de água. Sugere-se ainda acompanhar a sua comercialização no Brasil, de forma a verificar a provável exposição de seres humanos via consumo de água.

\section{SULFOXAFLOR}

O Sulfoxaflor foi autorizado no Brasil em dezembro de 2018, sendo um inseticida cuja aplicação foliar é permitida pela ANVISA em culturas de algodão, arroz, citros, feijão, melão, melancia, milho, soja, tomate e trigo (Agência Nacional de Vigilância Sanitária, 2019b). Nesse viés, cabe salientar que assim como o Dinotefuran e o Fluopiram, esse inseticida possui autorização para uso nas culturas de soja e milho, que são de relevância econômica para o Brasil. A aplicação do Sulfoxaflor deve ser feita quando polinizadores não estiverem nos arredores, pois o IA é altamente tóxico para as abelhas, como recomendado pelo Ministério da Saúde da Nova Zelândia (New Zealand, 2018).

De acordo com os dados expostos na Tabela 1, o valor do Koc para o Sulfoxaflor é 40,8 mL/g, indicando que o composto tem baixa capacidade de adsorção ao solo e, por isso, alta mobilidade nessa matriz (International Union of Pure and Applied Chemistry, 2019; Agency for Toxic Substances and Disease Registry, 1992). Além disso, o Sulfoxaflor apresenta baixo DT 50 no solo (2,2 dias), o que reforça a sua baixa persistência nessa matriz (Minnesota Department of Agriculture, 2013; International Union of Pure and Applied Chemistry, 2019). Por outro lado, o inseticida apresenta elevado DT50 na água (de 37 a 88 d), tendendo a permanecer nessa matriz (Minnesota Department of Agriculture, 2013; International Union of Pure and Applied Chemistry, 2019). Especificamente, o inseticida possui potencial de contaminação das águas subterrâneas baixo e das águas superficiais médio (Gustafson, 1989; Goss, 1992). Todavia, vale destacar que, tanto o índice GUS como o GOSS consideram o tempo de meia vida no solo em seus cálculos. Como o Sulfoxaflor tem um DT 50 no solo extremamente baixo, isso implica em um baixo potencial de contaminação das águas subterrâneas. No entanto, deve-se atentar para o médio potencial de contaminação para as águas superficiais, reforçado pela elevada 
solubilidade, baixo Koc e baixo Kow (ou seja, é um composto hidrofílico). Dessa forma, caso sejam utilizadas outras formas de aplicação que resultem no despejo do agrotóxico diretamente sobre a água (Ex.: pulverização acidental diretamente sobre um corpo hídrico), esse agrotóxico passa a ter relevância devido a sua elevada persistência na água. 0 escoamento superficial do Sulfoxaflor também pode ser uma rota importante na contaminação dos mananciais de abastecimento. Por fim, vale notar que a volatilidade desse composto é baixa, uma vez que a relação KH' /Kow é inferior ao valor de 10-9 e o KH' é menor que $10^{-4}$ (Rogers, 1996; Stenstrom et al., 1989). Na literatura consultada não foram encontrados estudos sobre a ocorrência do Sulfoxaflor em águas superficiais ou subterrâneas do Brasil.

O Sulfoxaflor não possui classificação carcinogênica pela International Agency for Research on Cancer (2019), porém a USEPA menciona que existem evidências sugestivas de potencial carcinogênico (United States Environmental Protection Agency, 2018b). O inseticida tem seu uso autorizado para aplicação em culturas em países/comunidades como Estados Unidos, Canadá, Austrália, Nova Zelândia e União Europeia (United States Environmental Protection Agency, 2019; Canada, 2019; Australian Pesticides and Veterinary Medicines Authority, 2013; New Zealand, 2018; European Food Safety Authority, 2019). Quanto à IDA, a Agência Nacional de Vigilância Sanitária (2019b) prevê um valor de 10,4 $\mu \mathrm{g} / \mathrm{kg}$ pc.d, sendo esse um valor mais restritivo quando comparado com aqueles indicados por agências internacionais como a USEPA, a EFSA, JMPR e a APVMA, que apresentam respectivamente IDA de 50,0 $\mu \mathrm{g} / \mathrm{kg}$ pc.d, 40,0 $\mu \mathrm{g} / \mathrm{kg}$ pc.d, 0 - 50,0 $\mu \mathrm{g} / \mathrm{kg}$ pc.d e 40,0 $\mu \mathrm{g} / \mathrm{kg}$ pc.d (United States Environmental Protection Agency, 2013; European Food Safety Authority, 2014b; Joint FAO/WHO Meeting on Pesticide Residues, 2011; Australian Pesticides and Veterinary Medicines Authority, 2013).

Considerando os estudos toxicológicos que embasam as IDA das agências internacionais, tem-se as doses experimentais de efeito adverso não observado (NOAEL) variando entre 4,24 e 5,13 mg/kg pc.d (Tabela 2), obtidas a partir de estudos crônicos com ratos (United States Environmental Protection Agency, 2013; European Food Safety Authority, 2014b; Joint FAO/WHO Meeting on Pesticide Residues, 2011; Australian Pesticides and Veterinary Medicines Authority, 2013). Em todos os estudos, a ingestão crônica de Sulfoxaflor resultou em hepatotoxicidade, alterações de peso e hipertrofia no fígado, alterações enzimáticas e degeneração hepatocelular (European Food Safety Authority, 2014b; Australian Pesticides and Veterinary Medicines Authority, 2013). Ressalta-se ainda a ocorrência de tumores hepáticos em estudo com ratos. Contudo, concluiu-se, nesse caso, risco potencial irrelevante para esse tipo de tumor em humanos (European Food Safety Authority, 2014b). Outros efeitos adversos reportados em estudos de exposição crônica com ratos incluem alterações no sistema reprodutivo (European Food Safety Authority, 2014b), distúrbios de desenvolvimento e morte de recém-nascidos (United States Environmental Protection Agency, 2013).

Possíveis valores-normativos para a concentração máxima de Sulfoxaflor em águas para o consumo humano variaram entre 62 e $308 \mu \mathrm{g} / \mathrm{L}$ (Tabela 2). 0 valor-normativo mais restritivo foi extraído com base na IDA reportada pela Agência Nacional de Vigilância Sanitária (2019b), cujos efeitos toxicológicos não foram especificados. Considerando a possibilidade de efeitos adversos ao fígado, tem-se valores-normativos de $254 \mu \mathrm{g} / \mathrm{L}$ (Australian Pesticides and Veterinary Medicines Authority, 2013; European Food Safety Authority, 2014b) e 308 g/L (Joint FAO/WHO Meeting on Pesticide Residues, 2011; United States Environmental Protection Agency, 2013) para água potável.

Com base no exposto, o Sulfoxaflor possui dinâmica ambiental desfavorável para contaminação das águas subterrâneas, todavia merece atenção sob o aspecto da elevada persistência na água e moderado potencial de contaminação das águas superficiais, bem como pelo fato de ser autorizado para uso em culturas importantes no Brasil, tais como soja e milho. Entretanto, o uso do agrotóxico nessas culturas deve ser confirmado pelos relatórios de comercialização de agrotóxicos no Brasil disponibilizados pelo IBAMA. Adicionalmente, são recomendados estudos de monitoramento do Sulfoxaflor em mananciais superficiais, onde o composto possui maior probabilidade de ocorrência.

Adicionalmente, por haver evidência sugestiva de ser um composto carcinogênico (United States Environmental Protection Agency, 2018b), hepatotóxico e causar efeitos reprodutivos, é recomendada atenção especial com relação a esse composto, sendo um possível candidato à regulamentação em normas de qualidade da água para consumo humano no Brasil. Reforça-se que nenhuma das agências/diretrizes consultadas recomenda um valor normativo/orientativo para a concentração do Sulfoxaflor em água. 


\section{CONCLUSÕES}

Entre janeiro de 2018 e dezembro de 2019, quatro ingredientes ativos de agrotóxicos foram autorizados para uso no Brasil (Dinotefuran, Florpirauxifen-benzil, Fluopiram e Sulfoxaflor) e 35 proibidos. Todos os compostos recentemente autorizados são utilizados em culturas de destaque no Brasil, como milho e soja, com exceção do Florpirauxifen-benzil.

Ainda que o Sulfoxaflor apresente elevada toxicidade e persistência ambiental, esse agrotóxico possui baixa mobilidade ambiental e, portanto, baixo potencial de contaminação dos mananciais de abastecimento de água, principalmente subterrâneo. No entanto, considerando o binômio toxicidade versus exposição utilizado na avaliação quantitativa de risco químico, julga-se relevante o monitoramento das vendas e ocorrência desse agrotóxico em sistemas de abastecimento de água, a fim de obter subsídios para uma possível regulamentação da concentração de tal composto em água de consumo humano. Já o Dinotefuran e o Fluopiram também possuem elevada toxicidade, no entanto, apresentam considerável persistência/mobilidade ambiental, sugerindo um maior risco do ponto de vista da exposição de seres humanos via consumo de água e uma possível necessidade de sua regulamentação no padrão de potabilidade da água brasileiro. No caso do Florpirauxifen-benzil, a sua baixa toxicidade e baixa mobilidade ambiental sugerem um menor risco da exposição de seres humanos via consumo de água e uma menor prioridade para monitoramento/regulamentação em água de consumo humano no Brasil.

Salienta-se ainda que uma possível revisão do padrão brasileiro de potabilidade da água deve ter um caráter mais amplo, abrangendo além dos novos agrotóxicos autorizados no período alvo desse estudo, aqueles que são autorizados e não constam na portaria, pautando-se em dados provenientes de estudos toxicológicos e dinâmica ambiental, a fim de identificar o potencial risco associado à saúde do indivíduo e contaminação de matrizes ambientais.

\section{AGRADECIMENTOS}

Os autores agradecem a Pró-Reitoria de Pós-Graduação e Pesquisa da Universidade Federal de Juiz de Fora (PROPP-UFJF) e ao CNPq pelas bolsas de Iniciação Científica concedidas.

\section{REFERÊNCIAS}

Agência Nacional de Vigilância Sanitária - ANVISA. (2019a). Reavaliação de agrotóxicos. Recuperado em 9 de dezembro de 2019, de http://portal.anvisa.gov.br/registros-eautorizacoes/agrotoxicos/produtos/reavaliacao-de-agrotoxicos

Agência Nacional de Vigilância Sanitária - ANVISA. (2019b). Registros e autorizações. Recuperado em 9 de dezembro de 2019, de http://portal.anvisa.gov.br/registros-eautorizacoes/agrotoxicos/produtos/monografia-de-agrotoxicos/autorizadas

Agência Nacional de Vigilância Sanitária - ANVISA. (2019c). Registros e autorizações. Recuperado em 9 de dezembro de 2019, de http://portal.anvisa.gov.br/registros-eautorizacoes/agrotoxicos/produtos/monografia-de-agrotoxicos/excluidas

Agência Nacional de Vigilância Sanitária - ANVISA. (2020). Registros e autorizações: painel de monografias de agrotóxicos. Recuperado em 29 de agosto de 2020, de https://app.powerbi.com/view?r=eyJrljoiMjBmMmM4ZDgtNTA5Yy00MWRiLTk2NjUtODYwM2JkMTY1Yzg xIiwidCI6ImI2N2FmMjNmLWMzZjMtNGQzNS04MGM3LWI3MDg1ZjVIZGQ4MSJ9

Agency for Toxic Substances and Disease Registry - ATSDR. (1992). ATSDR public health assessment guidance manual. Recuperado em 19 de dezembro de 2019, de https://books.google.com.br/books?id=eF6_mn0xcHcC\&pg=PA11\&lpg=PA11\&dq=atsdr++Koc\&source=bl\&ots=epvp8fxHHE\&sig=bUOZ4eosSMhpUVv64_H14XbvV20\&hl=ptBR\&sa=X\&ved=0ahUKEwiO24cDXAhVCGpAKHTF8BRQQ6AEISTAE\#v=onepage $\& \mathrm{q}=$ atsdr\%20$\% 20$ Koc $\& \mathrm{f}=$ false

Agency for Toxic Substances and Disease Registry - ATSDR. Toxic Substances Portal. (2019). Health effect of exposure to substances and carcinogens. Recuperado em 4 de dezembro de 2019, de https://www.atsdr.cdc.gov/substances/ToxOrganSystems.asp

Associação Brasileira de Saúde Coletiva - ABRASCO. (2015). Dossiê ABRASCO: um alerta sobre os impactos dos agrotóxicos à saúde. Rio de Janeiro: EPSJV; São Paulo: Escola Politécnica de Saúde Joaquim Venâncio: Expressão Popular. 
Australian Pesticides and Veterinary Medicines Authority - APVMA. (2013). Public Release Summary on the Evaluation of the New Active Constituent Sulfoxaflor in the Product Transform Insecticide. APVMA Product Number 64101. Recuperado em 6 de dezembro de 2019, de http://apvma.gov.au/sites/default/files/publication/14041-prs-sulfoxaflor.pdf

Australian Pesticides and Veterinary Medicines Authority - APVMA. (2015a). Public release summary on the evaluation of the new active constituent dinotefuran in the product transform insecticide: APVMA product number 63642. Recuperado em 6 de dezembro de 2019, de https://apvma.gov.au/sites/default/files/publication/18426-prs-dinotefuran.pdf

Australian Pesticides and Veterinary Medicines Authority - APVMA. (2015b). Public release summary on the evaluation of the new active constituent fluopyram in the product transform insecticide: APVMA product number 63642. Recuperado em 6 de dezembro de 2019, de https://apvma.gov.au/sites/default/files/publication/14166-prs-fluopyram.pdf

Australian Pesticides and Veterinary Medicines Authority - APVMA. (2018). Public Release summary on the evaluation of the new active constituent florpyrauxifen-benzyl in the product transform insecticide: APVMA product number 82885. Recuperado em 6 de dezembro de 2019, de https://apvma.gov.au/sites/default/files/publication/29096-gf-3301_herbicide-prs.pdf

Brasil. (1989, 12 de julho). Lei no 7.802, de 11 de julho de 1989. Dispõe sobre a pesquisa, a experimentação, a produção, a embalagem e rotulagem, o transporte, o armazenamento, a comercialização, a propaganda comercial, a utilização, a importação, a exportação, o destino final dos resíduos e embalagens, o registro, a classificação, o controle, a inspeção e a fiscalização de agrotóxicos, seus componentes e afins, e dá outras providências. Diário Oficial [da] República Federativa do Brasil, Brasília.

Brasil. (2002, 8 de janeiro). Decreto no 4.074, de 8 de janeiro de 2002. Regulamenta a Lei no 7.802, de 11 de julho de 1989, que dispõe sobre a pesquisa, a experimentação, a produção, a embalagem e rotulagem, o transporte, o armazenamento, a comercialização, a propaganda comercial, a utilização, a importação, a exportação, o destino final dos resíduos e embalagens, o registro, a classificação, o controle, a inspeção e a fiscalização de agrotóxicos, seus componentes e afins, e dá outras providências. Diário Oficial [da] República Federativa do Brasil, Brasília.

Brasil. Ministério da Saúde. (2017, 28 de julho). Portaria de Consolidação no 5, de 28 de setembro de 2017 ANEXO XX. Consolidação das normas sobre as ações e os serviços de saúde do Sistema Único de Saúde. Diário Oficial [da] República Federativa do Brasil, Brasília.

Cabral, A. R., Brandão, C. S., Grigoletto, J. C., Daniel, M. H. B., Bezerra, N. R., Bevilacqua, P. D., Bastos, R. K. X., \& Aquino, S. F. (2012). Documento base de elaboração da Portaria MS no 2.914/2011. "Portaria de Potabilidade da Água para Consumo Humano". Recuperado em 15 de janeiro de 2020, de http://portalarquivos2.saude.gov.br/images/pdf/2014/julho/24/Documento-Base-de-elaboracao-daPortaria-MS-2914.pdf.

Canada. Government of Canada. (2019). Sulfoxaflor. Recuperado em 19 de dezembro de 2019, de http://publications.gc.ca/site/eng/9.850784/publication.html

European Commission. (2003). Guidance Document for environmental risk assessments of active substances used on rice in the EU for annex I inclusion. Final Report of the Working Group "MED-RICE" prepared for the European Commission in the framework of Council Directive 91/414/EEC. SANCO/1090/2000-rev. 1.

European Food Safety Authority - EFSA. (2013). Conclusion on the peer review of the pesticide risk assessment of the active substance Fluopyram. EFSA Journal, 11(4), 3052-3128. http://dx.doi.org/10.2903/j.efsa.2013.3052

European Food Safety Authority - EFSA. (2014a). Regulation (EU) No 528/2012 concerning the making available on the market and use of biocidal products: Dinotefuran. Recuperado em 5 de dezembro de 2019, de <https://echa.europa.eu/documents/10162/e7b3136e-ddd2-9ac1-272c-3cd7949757f9

European Food Safety Authority - EFSA. (2014b). Conclusion on the peer review of the pesticide risk assessment of the active substance Sulfoxaflor. EFSA Journal, 12(5), 3692-3862. http://dx.doi.org/10.2903/j.efsa.2014.3692

European Food Safety Authority - EFSA. (2018). Conclusion on the peer review of the pesticide risk assessment of the active substance florpyrauxifen (variant assessed florpyrauxifen-benzyl). EFSA Journal, 16(8), 53785399. http://dx.doi.org/10.2903/j.efsa.2018.5378

European Food Safety Authority - EFSA. (2019). Recuperado em 5 de dezembro de 2019, de https://www.efsa.europa.eu/.

Goss, D. W. (1992). Screening procedure for soils and pesticides for potential water quality impacts. Weed Technology, 6(3), 701-708. http://dx.doi.org/10.1017/S0890037X00036083 
Gustafson, D. I. (1989). Groundwater ubiquity score: a simple method for assessing pesticide leachability. Environmental Toxicology and Chemistry, 8(4), 339-357. http://dx.doi.org/10.1002/etc.5620080411

Health Canada. (2017). Guideline for Canadian drinking water quality. Recuperado em 5 de dezembro de 2019, de https://www.canada.ca/en/health-canada/services/environmental-workplace-health/reportspublications/water-quality.html\#tech_doc

Instituto Brasileiro de Geografia e Estatística - IBGE. SIDRA. (2019). Banco de tabelas estatísticas. Recuperado em 19 de dezembro de 2019, de https://sidra.ibge.gov.br

International Agency for Research on Cancer - IARC. (2019). Agents classified by the IARC monographs (Vol. 1125). Recuperado em 5 de dezembro de 2019, de https://monographs.iarc.fr/agents-classified-by-the-iarc/

International Union of Pure and Applied Chemistry - IUPAC. (2019). Pesticide properties database. Recuperado em 20 de dezembro de 2019, de https://sitem.herts.ac.uk/aeru/iupac/

Joint FAO/WHO Meeting on Pesticide Residues - JMPR. (2010). Fluopyram. Recuperado em 4 de dezembro de 2019, de https://apps.who.int/pesticide-residues-jmpr-database/pesticide?name=FLUOPYRAM

Joint FAO/WHO Meeting on Pesticide Residues - JMPR. (2011). Sulfoxaflor. Recuperado em 15 de dezembro de 2019, de https://apps.who.int/pesticide-residues-jmpr-database/pesticide?name=SULFOXAFLOR

Joint FAO/WHO Meeting on Pesticide Residues - JMPR. (2012). Dinotefuran. Recuperado em 4 de dezembro de 2019, de <https://apps.who.int/pesticide-residues-jmpr-database/pesticide?name=DINOTEFURAN

Minnesota Department of Agriculture - MDA. (2012). Fluopyram. New Active Ingredient Review. Minnesota: MDA.

Minnesota Department of Agriculture - MDA. (2013). Sulfoxaflor. New Active Ingredient Review. Minnesota: MDA.

Minnesota Department of Agriculture - MDA. (2018). Florpyrauxifen-benzyl. New Active Ingredient Review. Minnesota: MDA.

National Health and Medical Research Council - NHMRC. Natural Resource Management Ministerial Council NRMMC. (2018). Australian Drinking Water Guidelines 6: version 3.5 Updated August 2018. Recuperado em 5 de dezembro de 2019, de https://nhmrc.gov.au/about-us/publications/australian-drinking-waterguidelines\#block-views-block-file-attachments-content-block-1

National Pesticide Information Center - NPIC. (2019). OSU extension pesticide properties database. Recuperado em 16 de dezembro de 2019, de http://npic.orst.edu/ingred/ppdmove.htm

New Zealand. Ministry of Health. (2018). Guidelines for drinking-water quality management for New Zealand: Datasheets part 2.1: chemical and physical determinands. Recuperado em 5 de dezembro de 2019, de https://www.health.govt.nz/publication/guidelines-drinking-water-quality-management-new-zealand

Pereira, R. O., Assunção, T. O. G., Dias, A. C. L., Gomes, F. B. R., \& Brandt, E. M. F. (2019). Estudo da comercialização de agrotóxicos listados no padrão de potabilidade da água de consumo humano no Brasil In Anais do XXX Congresso Brasileiro de Engenharia Sanitária e Ambiental. Natal.

Rice, P. J., Rice, P. J., Arthur, E. L., \& Barefoot, A. C. (2007). Advances in pesticide environmental fate and exposure assessments. Journal of Agricultural and Food Chemistry, 55(14), 5367-5376. http://dx.doi.org/10.1021/jf063764s

Rogers, H. R. (1996). H. R. Sources, behaviour and fate of organic contaminants during sewage treatment and in sewage sludges. The Science of the Total Environment, 185(1-3), 3-26. http://dx.doi.org/10.1016/00489697(96)05039-5

Staudinger, J., \& Roberts, P. V. (1996).A critical review of Henry's law constants forenvironmental applications. Critical Reviews in Environmental Science and Technology, 26(3), 205-297. http://dx.doi.org/10.1080/10643389609388492

Stenstrom, M. K., Cardinal, L., \& Libra, J. (1989). Treatment of hazardous substances in wastewater treatment plants. Environment and Progress, 8(2), 107-112. http://dx.doi.org/10.1002/ep.3300080214

United States Environmental Protection Agency - USEPA. (2004). Agency dinotefuran pesticide fact sheet. Recuperado em 10 de dezembro de 2019, de https://www3.epa.gov/pesticides/chem_search/reg_actions/registration/fs_PC-044312_01-Sep-04.pdf

United States Environmental Protection Agency - USEPA. (2013). Pesticide tolerances: sulfoxaflor. Recuperado em 5 de dezembro de 2019, de https://www.regulations.gov/document?D=EPA-HQ-OPP-2010-0889-0402 
United States Environmental Protection Agency - USEPA. (2018a). 2018 edition of the drinking water standards and health advisories. Recuperado em 5 de dezembro de 2019, de https://www.epa.gov/dwstandardsregulations/2018-drinking-water-standards-and-advisory-tables

United States Environmental Protection Agency - USEPA. (2018b). Chemicals evaluated for carcinogenic potential annual cancer report. Recuperado em 17 de dezembro de 2019, de http://npic.orst.eu/chemicals_evaluated.pdf

United States Environmental Protection Agency - USEPA. (2019). Pesticide tolerances: fluopyram. Recuperado em 5 de dezembro de 2019, de https://www.federalregister.gov/documents/2019/07/01/201913523/fluopyram-pesticide-tolerances

United States Environmental Protection Agency - USEPA. (2020). Overview of risk assessment in the Pesticide Program. Recuperado em 8 de janeiro de 2020, de https://www.epa.gov/pesticide-science-and-assessingpesticide-risks/overview-risk-assessment-pesticide-program

Valarini, P. J., Nardo, E. A. B., Gomes, M. A. F., Ferreira, C. J. A., \& Castro, V. L. S. S. (1998). Dinâmica e efeitos de agrotóxicos no meio ambiente. In: F. C. O. Freire, J. E. Cardoso \& F. M. P. Viana (Orgs.), Doenças de fruteiras tropicais de interesse agroindustrial (pp. 625-686). Brasília: Embrapa-SPI.

Veiga, M. M. (2007). Agrotóxicos: eficiência econômica e injustiça socioambiental. Ciencia \& Saude Coletiva, 12(1), 145-152. http://dx.doi.org/10.1590/S1413-81232007000100017

World Health Organization - WHO. (2017). Guidelines for drinking-water quality: fourth edition incorporating the first addendum 5 ed. Recuperado em 12 de dezembro de 2019, de http://www.who.int/water_sanitation_health/publications/drinking-water-quality-guidelines-4-including1st-addendum/en/

\section{Contribuições dos autores:}

Taciane de Oliveira Gomes de Assunção: Contribuição na concepção da nota técnica, revisão de literatura, análise dos dados e escrita do documento.

Fernanda Bento Rosa Gomes: Contribuição na concepção da nota técnica, revisão de literatura, análise dos dados e escrita do documento.

Emanuel Manfred Freire Brandt: Contribuição na concepção da nota técnica, orientação e revisão.

Renata de Oliveira Pereira: Contribuição na concepção da nota técnica, orientação e revisão. 